\title{
La co-producción de la Naturaleza Urbana: resultados del I Taller de Naturalezas Híbridas
}

Diz, Carlos; Santiago-Gómez, Elvira; Cortés-Vázquez, José A.

Facultade de Socioloxía, Universidade da Coruña.

\section{RESUMEN}

El "I Taller de Naturalezas Híbridas" tuvo como objetivo explorar nuevas formas de aprendizaje activo, interdisciplinar y colaborativo en el estudio de temas complejos para el alumnado. En esta edición nos centramos en las "naturalezas urbanas" como concepto y campo de estudio a partir del que problematizar los límites y fronteras de conocimiento dentro de la sociología. Integrando interdisciplinarmente las Ciencias Sociales, las Ciencias Medioambientales, la Arquitectura y las Ciencias de la Comunicación, las estudiantes pudieron analizar la relación entre naturaleza, sociedad y ciudad a través de espacios urbanos liminales y periféricos, donde lo natural, lo social y lo urbano se encuentran entrelazados e "hibridados". Para ello organizamos una jornada de debate-ruta-debate que tuvo como destino varios espacios "híbridos" de A Coruña. El alumnado pudo reflexionar, desde el terreno, sobre espacios donde se plasman representaciones que superan la división entre lo urbano y lo natural como dominios separados. Esto incluía parques y espacios ubicados en intersticios urbanos y que son hogar de ontologías híbridas que ponen en entredicho esa separación entre lo urbano, lo natural y lo social. Se logró con ello un aprendizaje más rico, diverso y complejo, lo que nos anima a repetir la experiencia en cursos venideros.

PALABRAS CLAVE: Naturaleza, Hibridación, Colaboración, Co-producción, Aprendizaje activo. 


\section{CITA RECOMENDADA:}

Diz, C.; Santiago-Gómez, E. ; Cortés-Vázquez, José A. (2019): La co-producción de la Naturaleza Urbana: resultados del I Taller de Naturalezas Híbridas. En De la Torre Fernández, E. (ed.) (2019). Contextos universitarios transformadores: construíndo espazos de aprendizaxe. III Xornadas de Innovación Docente. Cufie. Universidade da Coruña. A Coruña (pág. 333-344).

DOI capítulo: https://doi.org/10.17979/spudc. 9788497497121.333

DOl libro: https://doi.org/10.17979/spudc.9788497497121

\section{ABSTRACT}

The "1st Workshop on Hybrid Natures" aimed to explore new forms of active, interdisciplinary and collaborative learning in the study of complex issues for students. In this edition, we focus on the "urban natures" as a concept and field of study from which to problematize the limits and frontiers of knowledge within sociology. In an integrative and interdisciplinary approach of the Social Sciences, Environmental Sciences, Architecture and Communication Sciences, the students were able to analyze the relationship between nature, society and city through liminal and peripheral urban spaces, where the natural, the social and urban are intertwined and "hybridized". For this, we organized a day of debateroute-debate that had as destination several "hybrid" spaces of A Coruña. The students were able to reflect, from the field, on spaces where representations overcome the division between the urban and the natural as separate domains. This included parks and spaces located in urban interstices and that are home to hybrid ontologies that call into question that separation between the urban, the natural and the social. This resulted in a richer, more diverse and more complex learning, which encourages us to repeat the experience in future courses.

KEY WORDS: Nature, Hybridization, Collaboration, Co-production, Active Learning. 


\section{INTRODUCCIÓN}

La actividad "I Taller de Naturalezas Híbridas" parte de tres PDI de la Facultad de Sociología con especialidades distintas (estudios ambientales, estudios urbanos, seguridad y vigilancia) pero con una motivación compartida: trazar puentes interdisciplinares, tanto a nivel investigador como docente, y explorar estrategias más participativas para abordar el estudio y comprensión de temas de especial complejidad. Nuestro objetivo principal con este taller era diseñar una herramienta de innovación docente que pudiéramos replicar cada curso académico y que nos permitiera hacer frente a dos retos clave: a) plantear soluciones a la híper-especialización de la carrera investigadora, puesto que supone una barrera para abordar temas generales desde la docencia; y b) la necesidad de desarrollar dinámicas docentes más simétricas, donde la enseñanza se entienda como orientación y guía, promoviendo la coconstrucción del conocimiento (Monereo y Pozo, 2003; González Tirado y González Maura, 2007).

Cabe destacar que esta idea de co-construcción viene encuadrada dentro del "giro colaborativo" que en la última década, y especialmente en el campo de las ciencias sociales, se viene produciendo con especial intensidad (Holmes y Marcus, 2008; Estalella y SánchezCriado, 2018). Este giro replantea los límites tradicionales del saber experto y anima al ensayo y a la experimentación -entre docentes, estudiantes e investigadores- de nuevas fórmulas pedagógicas y metodológicas con las que encarar los retos epistemológicos ante los que nos sitúa la sociedad y la universidad del siglo XXI. Así, nos invita a repensar nuestros repertorios de acción y a imaginar otras maneras de hacer y trabajar en común.

La idea de "Naturalezas Híbridas" sobre la que gira este taller hace referencia a una de las cuestiones de mayor complejidad para la docencia de la disciplina sociológica: la relación entre los conceptos de sociedad, naturaleza y ciudad; campos en los que las tres PDI han desarrollado sus distintas especializaciones investigadoras. Para esta primera edición del taller, y de cara a cumplir con los objetivos antes señalados, planteamos organizar conjuntamente un debate-ruta-debate dirigido al alumnado de Sociología y Ciencias de la 
Comunicación y a la comunidad docente e investigadora del departamento, centrado en la coproducción de conocimiento sobre la naturaleza urbana de A Coruña. Nos centramos, sobre todo, en aquellos espacios donde esta naturaleza urbana aparece más difusa (los márgenes de la ciudad) de modo que pudiéramos entrar en contacto directo con espacios teoréticamente problemáticos para la disciplina sociológica.

La idea de centrarnos en las naturalezas urbanas fue clave porque obligaba a las estudiantes a intentar entender y explicar no solo aquellos espacios donde se plasman representaciones que superan la clásica división entre "Io urbano" y "Io natural" como dominios separados -como es el caso de los parques periurbanos-, sino también los espacios "liminales" (solares, casas abandonadas, caminos sin asfaltar, estanques, cunetas o lagunas) que, a distintas escalas, se desarrollan en los intersticios urbanos y son hogar de ontologías híbridas y múltiples que ponen en entredicho esa separación entre lo urbano, lo natural y lo social. Hablamos, por lo tanto, de entes híbridos, que retan así las mismas divisiones del conocimiento experto entre disciplinas aplicadas sobre ámbitos de estudio supuestamente purificados de estas hibridaciones. He ahí que para su análisis y reflexión se requieran acercamientos igualmente híbridos (es decir, interdisciplinares, mestizos y colaborativos), que buscamos co-generar y co-producir en el diálogo entre estudiantes, investigadores y docentes. Así pues, la hibridación, al inicio de esta propuesta de innovación, no era solo un objeto de indagación desplegado sobre el espacio urbano, sino que tenía también un carácter recursivo: la hibridación se planteaba a su vez como una vía para repensar nuestras prácticas académicas.

De este modo, El "I Taller de Naturalezas Híbridas: Co-producción de la naturaleza urbana" cumplía con el propósito inicial de plantear una aproximación interdisciplinar entre las Ciencias Sociales, las Ciencias Medioambientales y las Ciencias de la Comunicación a la relación entre naturaleza, sociedad y ciudad, a la vez que generaba un espacio colaborativo entre los participantes (estudiantes, investigadores y docentes) en el que co-producir reflexiones, interpretaciones y saberes de Naturalezas Urbanas que sirvan para poner el foco sobre la 
recurrencia de procesos de hibridación en el seno de lo que podríamos entender como espacios normativos o normativizados (el espacio puramente urbano, el espacio puramente natural); lo que nos lleva a terrenos más amplios de debate sobre la construcción de estos espacios y la función del mundo académico en su reproducción 0 en su cuestionamiento crítico.

\section{DESCRIPCIÓN DE LA EXPERIENCIA}

El I Taller de Naturalezas Híbridas se celebró el 4 de octubre de 2018, coincidiendo con el Día Nacional de las Universidades Saludables. La actividad formó parte del programa docente de las asignaturas del Grado en Sociología: Globalización, medioambiente y población y Riesgo, cultura y medioambiente, y se abrió a la participación del conjunto de la comunidad universitaria. Para su organización se contó con una ayuda económica concedida por el Departamento de Sociología y Ciencias de la Comunicación, la cual se empleó para cubrir los gastos de participación del ponente invitado, el arquitecto y urbanista lago Carro, que en la última década ha emprendido numerosas investigaciones sobre lo urbano y sus naturalezas en el ámbito coruñés, miembro de la Asociación Cultural Ergosfera y de Partidarios da Cidade Sociedade Cooperativa Galega.

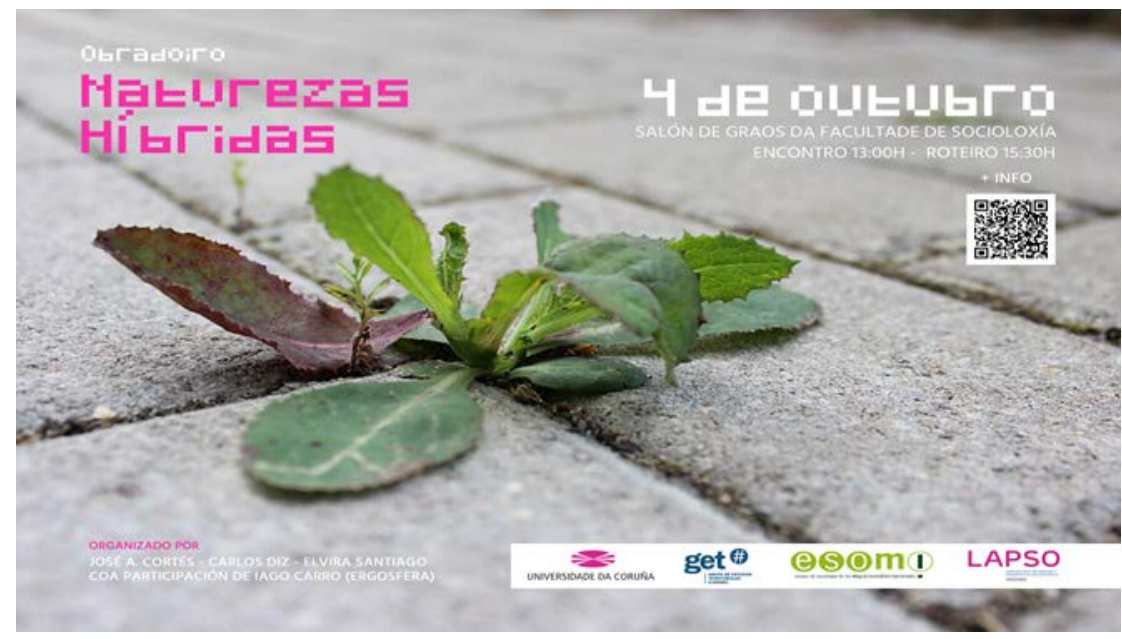

Imagen 1. Cartel del I Taller de Naturalezas Híbridas 
El Taller se estructuró en tres bloques. Tras una pequeña presentación en el Salón de Grados de la Facultad de Sociología, la primera parte consistió en una charla a cargo de lago Carro, que desde su amplia experiencia en el ámbito de la investigación urbana aplicada invitó a la reflexión teórica sobre una serie de conceptos y preguntas clave: ¿Qué tipos de naturalezas encontramos en la ciudad y dónde se materializan? ¿Cómo se organiza la (bio)diversidad urbana? ¿Cómo se generan política y culturalmente los espacios normativos de lo urbano y lo natural? ¿Qué es un "espacio verde"? ¿Qué funciones sociales cumplen las distintas naturalezas de una ciudad? ¿Qué son y cómo se gobiernan las naturalezas híbridas metropolitanas? ¿Qué grado de participación y decisión tenemos en el diseño de nuestras ciudades? ¿Cuál es la naturaleza de lo urbano?

La segunda parte del taller fue la más extensa y aplicada. Comenzó a las 15:00 horas con el inicio de la ruta, que discurrió desde la entrada principal de la Facultad de Sociología, en el Campus de Elviña, hasta la llegada al Puerto de Bens a las 20:30 horas, pasando por los diferentes espacios híbridos y reflexionando conjuntamente y en el terreno sobre sus significados. A lo largo de $10 \mathrm{~km}$ de caminata, el grupo de estudiantes, docentes e investigadores se fue deteniendo en los lugares más significativos propuestos por el experto invitado, discutiendo las propuestas teóricas comentadas en la sesión matinal y viendo su aplicación directa en el entorno coruñés: las cuencas de los ríos Mesoiro y Monelos, la laguna natural-artificial de Someso, el corredor verde que se extiende sobre el oleoducto de la ciudad, el parque de Vioño, San Pedro de Visma, Bens y 0 Portiño fueron algunos de los lugares visitados, caminados y discutidos colectivamente a lo largo de la jornada.

La tercera parte se refiere a las reflexiones que tuvieron lugar al terminar la ruta, tanto inmediatamente después durante el coloquio que dio cierre a la prolongada caminata -en un intercambio jovial y aireado que tuvo lugar en un escenario distinto al de las aulas- como a través de los espacios virtuales que fueron generados durante el taller para el intercambio de información, comentarios, fotografías, vídeos y documentos escritos. Habiendo creado previamente un código QR para la subida y el almacenamiento de información en la web -en 
Google Drive, más concretamente-, a posteriori el alumnado fue subiendo sus reflexiones, a la vez que durante la jornada -en tiempo real- subían y comentaban sus impresiones en redes sociales (Instagram, WhatsApp, Facebook y Twitter). De este modo, el proceso de hibridación también se iba produciendo, sobre la marcha y de un modo colectivo, en la combinación de la forma analógica con la digital. El propio proceso, la jornada, se iba nutriendo paulatinamente de sucesivas hibridaciones.

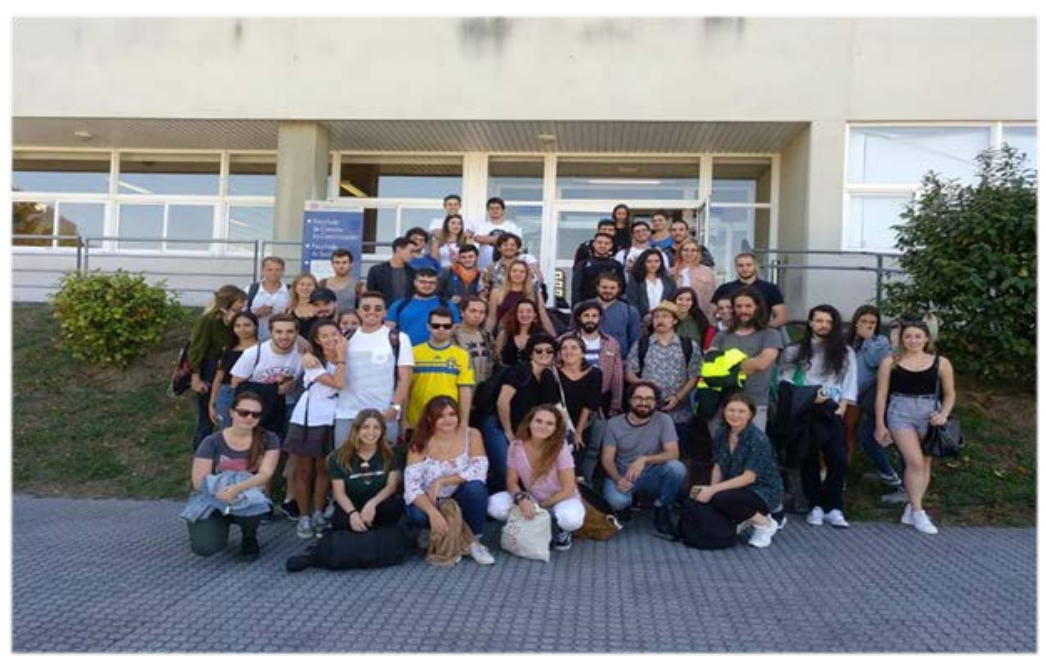

Imagen 2. Inicio de la ruta

\section{RESULTADOS}

Los principales resultados del taller fueron:

1. Colaboración docente interdisciplinar entre PDI con distintas especializaciones y radicadas en distintos grupos de investigación (ESOMI- Equipo de Sociología de las Migraciones Internacionales; GET- Grupo de Estudios Territoriales; LAPSO- Grupo de Análisis y Prospectiva Sociopolítica), con el objetivo de facilitar el acercamiento del alumnado de sociología a temáticas complejas y fundamentales que requieren de visiones transversales. 
2. Uso de formato de aprendizaje activo, basado en un primer planteamiento de las bases teóricas a discutir y su relevancia para la disciplina, y una posterior aproximación práctica a la complejidad de estos conceptos mediante la experiencia directa con aquellos espacios que rompen las barreras teóricas y epistemológicas de la sociología. Se lograba así que los estudiantes tuvieran la oportunidad de experimentar directamente las barreras que supone la división entre disciplinas académicas y su posterior híper-especialización intradisciplinaria para la comprensión de mundos complejos. Igualmente, se lograba que entrelazaran esas experiencias con otras más cotidianas de su día a día (al ser los espacios visitados también espacios que transitan de forma habitual e incluso diaria), de modo que les permitiera replicar el replanteamiento generado durante el taller en otros momentos y contextos.

3. Apuesta por procesos de enseñanza-aprendizaje saludables. Junto al sentido práctico y aplicado de la actividad, su valor saludable quedó patente en su inclusión en el Outubro UDC Saudable 2018, pues el taller fue realizado en el Día Nacional de las Universidades Saludables. Sacar al alumnado (y también a los docentes) de las aulas, invitarlos a recorrer a pie su ciudad y conectar el cuerpo con el entorno y el territorio, supuso una manera saludable y agradable de airear el pensamiento y la Academia; el I Taller de Naturalezas Híbridas enfatizó así el valor dinámico, experimental y peripatético del aprendizaje, reforzando un pensamiento caminado, en movimiento y en acción, y enredando el carácter experimental de esta colaboración docente con la necesidad de experimentar con todos los sentidos (en) los procesos de conocimiento.

4. Producción de documentos y archivos abiertos (y en abierto). La necesidad de airear las reflexiones fue de la mano del deseo de hacerlas públicas. Así se generaron plataformas virtuales donde cada estudiante iba subiendo sus comentarios, anotaciones, pensamientos y creaciones (documentos, vídeos, etc.). A pesar de las dificultades, el taller logró co-producir imágenes, vídeos y documentos escritos de 
docentes y estudiantes. Se generó una carpeta colectiva donde cada uno podía subir sus materiales y visualizar los de las demás. Aunque inacabado y lejos de la perfección, este proceso de archivo y documentación enriqueció el debate sobre la co-producción de conocimientos y la necesidad de abrir la universidad y sus saberes al conjunto de la ciudadanía, haciéndolos así replicables y reapropiables.

5. Ensamblaje e hibridación de formas analógicas y digitales de experimentación. A la vez que las estudiantes e investigadoras tomaban notas en sus cuadernos o hacían preguntas durante la charla y la ruta, también subían fotos al Instagram 0 al Facebook, retwitteaban los mensajes más populares o compartían la información a través de las redes sociales, por ejemplo usando el hashtag recomendado por la Universidade da Coruña (\#DiaUniSaludables) para hacer más visible en el ciberespacio la promoción de la salud en la UDC. La apuesta de los PDI por el uso (e invitación al uso) de las TIC se mostró de utilidad durante la jornada, a la vez que se vio superada y mejorada por el amplio manejo, destreza y creatividad de las estudiantes a la hora de expresarse digitalmente.

\section{CONCLUSIONES}

La experiencia de innovación docente "I Taller de Naturalezas Híbridas: la co-producción de la naturaleza urbana" perseguía el ambicioso proyecto de superar las barreras de la híperespecialización investigadora y del aprendizaje unidireccional y dirigido, suplantándolo con estrategias multi-disciplinares y colaborativas y formas de aprendizaje práctico, directo, experiencial y de descubrimiento. La consecución de ambos objetivos nos anima a repetir la experiencia en cursos futuros, incorporando nuevas rutas, nuevas preguntas y otros entornos de aprendizaje. Sin embargo, para ello -y para serle fieles a la experiencia de este primer taller- debemos tener en cuenta aquellos aspectos tanto negativos como positivos que surgieron durante su desarrollo. 
En cuanto a aspectos negativos, debemos señalar la dificultad de cuadrar los horarios de los tres PDI para encontrar un hueco lo suficientemente amplio como para desarrollar una actividad de varias horas. También debemos señalar la dificultad de cuadrar los horarios de los estudiantes, así como la dificultad de que estos y estas participen en actividades propuestas fuera del horario lectivo. Al ser una actividad vinculada a dos asignaturas distintas con horario diferente, tuvimos que buscar la manera de que no se solapara con otras asignaturas. Al ser una actividad en parte desarrollada al aire libre y en previsión de contar con condiciones atmosféricas favorables, elegimos llevarla a cabo en la primera mitad del curso (mes de octubre), cuando los estudiantes aún no han alcanzado el óptimo de profundización en los aspectos teóricos de las dos materias en las que el taller se enmarcaba.

En cuanto a los aspectos positivos, destacó el interés por parte de las estudiantes de distintos cursos -cuya asistencia desbordó lo esperado-, al ser un tema transversal en relación con el conjunto de la disciplina, y habiendo participado durante la jornada no solo las alumnas de las dos materias mencionadas sino un buen número de estudiantes de otros cursos que acudieron voluntariamente a la charla y a la caminata. Al tener un carácter aplicado, activo y experiencial, se logró aumentar el interés y facilitar una forma de aprendizaje más amena y autodirigida, y que se puede poner en relación con experiencias cotidianas extra-académicas. El éxito de la convocatoria generó además un interés por dar continuidad a la experiencia abriéndola a nuevos espacios, temas y contextos.

\section{REFERENCIAS}

Estalella, A. \& Sánchez-Criado, T. (Eds.). (2018). Experimental Collaborations: Ethnography through Fieldwork Devices. New York: Berghahn Books.

González Tirados, R. M., \& González Maura, V. (2007). Diagnóstico de necesidades y estrategias de formación docente en las universidades. Revista Iberoamericana de Educación 43(6), pp. 1-14. 
Holmes, D., \& Marcus, G. (2008). Collaboration Today and the Re-Imagination of the Classic Scene of Fieldwork Encounter. Collaborative Anthropologies 1(1), pp. 81-101.

Monereo, C., \& Pozo, J. I. (2003) La cultura educativa en la universidad: nuevos retos para profesores y alumnos. En C. Monereo \& J.I. Pozo (Eds.) La universidad ante la nueva cultura educativa: enseñar y aprender para la autonomía ). Madrid: Síntesis, pp. 15-32. 
